\title{
Bortezomib alleviates drug-induced liver injury by regulating CYP2E1 gene transcription
}

\author{
WOO-JAE PARK ${ }^{1 *}$, SO-YEON KIM ${ }^{2 *}$, YE-RYUNG KIM ${ }^{2}$ and JOO-WON PARK ${ }^{2}$ \\ ${ }^{1}$ Department of Biochemistry, School of Medicine, Gachon University, Incheon 406-799; ${ }^{2}$ Department of Biochemistry, \\ School of Medicine, Ewha Womans University, Yang Cheon-Gu, Seoul 158-710, Republic of Korea
}

Received May 7, 2015; Accepted January 14, 2016

DOI: 10.3892/ijmm.2016.2461

\begin{abstract}
Acute liver failure, i.e., the fatal deterioration of liver function, is the most common indication that emergency liver transplantation is necessary. Moreover, in the USA, drug-induced liver injury (DILI), including acetaminophen (APAP)-induced hepatotoxicity, is the main cause of acute liver failure. Matching a donor for liver transplantation is extremely difficult, and thus the development of a novel therapy for DILI is urgently needed. Following recent approval by the FDA of the proteasomal inhibitor bortezomib, its therapeutic effects on various human diseases, including solid and hematologic malignancies, have been validated. However, the specific action of proteasomal inhibition in cases of DILI had not been elucidated prior to this study. To examine the effects of proteasomal inhibition in DILI experimentally, male $\mathrm{C} 56 \mathrm{Bl} / 6$ mice were injected with $1 \mathrm{mg}$ bortezomib/kg before APAP treatment. Bortezomib not only alleviated APAP-induced hepatotoxicity in a time- and dose-dependent manner, it also alleviated $\mathrm{CCl}_{4}$ - and thioacetamide-induced hepatotoxicity. We also noted that bortezomib significantly reduced cytochrome P450 2E1 (CYP2E1) expression and activity in the liver, which was accompanied by the
\end{abstract}

Correspondence to: Professor Joo-Won Park, Department of Biochemistry, School of Medicine, Ewha Womans University, 911-1 Mok-Dong, Yang Cheon-Gu, Seoul 158-710, Republic of Korea E-mail: joowon.park@ewha.ac.kr

*Contributed equally

Abbreviations: DILI, drug-induced liver injury; ALT, alanine aminotransferase; APAP, acetaminophen; 2-APB, 2-aminoethyl diphenylborinate; AST, aspartate aminotransferase; BiP, binding immunoglobulin protein; CHOP, CCAAT-enhancer-binding protein homologous protein; CX, connexin; DMEM, Dulbecco's modified Eagle's medium; ER, endoplasmic reticulum; FDA, US Food and Drug Administration; GAPDH, glyceraldehyde-3-phosphate dehydrogenase; GSH, glutathione; HNF, hepatocyte nuclear factor; NAPQI, N-acetyl-p-benzoquinone imine; 4-PBA, sodium 4-phenylbutyrate; TAA, thioacetamide

Key words: bortezomib, liver, cytochrome P450 2E1, toxicity, acetaminophen, proteasome induction of endoplasmic reticulum (ER) stress. In addition, bortezomib decreased hepatocyte nuclear factor- $1 \alpha$-induced promoter activation of CYP2E1 in Hep3B cells. By contrast, another proteasome inhibitor, MG132, did not cause ER stress and did not markedly affect CYP2E1 enzyme activity. Liver injury induced by APAP was aggravated by MG132, possibly via elevation of connexin 32 expression. This study suggests that proteasome inhibition has different effects in cases of DILI depending on the specific inhibitor being used. Furthermore, results from the mouse model indicated that bortezomib, but not MG132, was effective in alleviating DILI. ER stress induced by proteasome inhibition has previously been shown to exert various effects on DILI patients, and thus each available proteasomal inhibitor should be evaluated individually in order to determine its potential for clinical application.

\section{Introduction}

Drug-induced liver injury (DILI) is the most frequent cause of acute liver failure (1); of various drugs, acetaminophen (APAP) overdose accounts for approximately one-half of all cases of acute liver failure in the USA and the UK (2). Acute liver failure suddenly affects young, otherwise healthy individuals, and is associated with an extremely high rate of mortality, and is the most frequent cause of emergency liver transplantation (1). Drug-induced hepatotoxicity is usually caused by the formation of drug metabolites, which are often formed by the action of cytochrome P450 2E1 (CYP2E1; EC 1.14.13.n7), which transforms various chemicals into reactive metabolites. For example, APAP is converted by CYP2E1 into $\mathrm{N}$-acetyl-p-benzoquinoneimine (NAPQI), an electrophilic metabolite that binds to cysteine groups in proteins (3), depletes glutathione (GSH), and causes respiratory dysfunction and reactive oxidative stress (4). Since finding a matching donor for liver transplantation is not always easy, novel therapies for treating DILI are urgently needed.

The proteasome is a multisubunit enzyme complex that degrades ubiquitin-tagged proteins; it plays a critical role in the regulation of proteins that control cell-cycle progression and apoptosis. Consequently, it has become an important target for anticancer therapy (5). In in vitro experiments and in animal studies, the inhibition of the proteasome, either alone or in combination with conventional chemotherapeutic agents, demonstrated antitumour effects against numerous 
tumour types (5). In 2008, bortezomib (VELCADE; formerly, PS-341, LDP-341, and MLN341) was approved by the US Food and Drug Administration (FDA) as a therapeutic agent for multiple myeloma (6). Recently, the therapeutic potential of bortezomib has been re-evaluated, and it has been reported that the compound is therapeutically effective for various diseases, such as tumours (7), congenital erythropoietic porphyria (8), and graft-versus-host disease (9).

To investigate the role of the proteasome and the effects of its inhibition on DILI, we examined the effects of two proteasome inhibitors, bortezomib and MG132, on drug- and chemicalinduced hepatotoxicity. Interestingly, bortezomib alleviated APAP-induced hepatotoxicity, whereas MG132 had the opposite effect. In addition, bortezomib treatment decreased liver damage induced by $\mathrm{CCl}_{4}$ or thioacetamide (TAA) and significantly decreased hepatic CYP2E1 transcription, leading to diminished enzyme activity. Results of the present study suggest that clinical treatment with bortezomib may be useful for alleviating DILI and possibly other forms of acute liver disease.

\section{Materials and methods}

Materials. Bortezomib was purchased from Biovision (Mountain View, CA, USA). APAP, $\mathrm{CCl}_{4}$, TAA, MG132, sodium 4-phenylbutyrate (4-PBA), and 2-aminoethyl diphenylborinate (2-APB) were purchased from Sigma-Aldrich (St. Louis, MO, USA). The primary antibodies used in this study were anti-CYP2E1 (AB1252; Millipore, Bedford, MA, USA), anticonnexin 32 (CX32; 35-8900; Invitrogen, Carlsbad, CA, USA), anti-glyceraldehyde-3-phosphate dehydrogenase (GAPDH; MAB374) (Millipore, Billerica, MA, USA), anti-binding immunoglobulin protein (BiP; 3177; Cell Signaling Technology, Beverly, MA, USA), anti-CCAAT-enhancer-binding protein homologous protein (CHOP; 5554; Cell Signaling Technology) and anti- $\beta$-actin (A5316; Sigma-Aldrich).

Animals. Male C57BL/6J mice, which were 6-8 weeks of age, were purchased from Orient Bio, Inc. (Seoul, Korea) and housed under special pathogen-free conditions. All animals were treated in accordance with the Animal Care Guidelines of Ewha Womans University. To induce hepatotoxicity, mice were injected intraperitoneally with the following: APAP (350 or $500 \mathrm{mg} / \mathrm{kg})$, TAA $(200 \mathrm{mg} / \mathrm{kg})$, or $\mathrm{CCl}_{4}(2 \mathrm{ml} / \mathrm{kg})$, as described previously (10). Before being injected, mice were fasted overnight. To inhibit the proteasome, bortezomib was injected twice: first at $12 \mathrm{~h}(1 \mathrm{mg} / \mathrm{kg})$ and then at $1 \mathrm{~h}(1 \mathrm{mg} / \mathrm{kg})$ prior to the injection of APAP, as previously described (11). The inhibitor MG132 was injected $(5 \mathrm{mg} / \mathrm{kg})$ twice: first at $12 \mathrm{~h}$ and then at $1 \mathrm{~h}$ prior to the administration of APAP, as previously described $(12,13)$. In some cases, different doses of bortezomib $(0-1 \mathrm{mg} / \mathrm{kg})$ were injected twice: first at $12 \mathrm{~h}$ and then at $1 \mathrm{~h}$ prior to the administration of APAP, and $1 \mathrm{mg} / \mathrm{kg}$ bortezomib was injected at different times prior to the administration of APAP. For the inhibition of gap junctions, 2-APB $(20 \mathrm{mg} / \mathrm{kg})$ was injected $2 \mathrm{~h}$ prior to the administration of APAP. DMSO-treated mice were used as the relevant control group. Each group consisted of 4-6 mice. For liver extraction, the mice were sacrificed at $0,2,46 \mathrm{~h}$ after the APAP injection and, at $24 \mathrm{~h}$ after the TAA or $\mathrm{CCl}_{4}$ injection. The livers were then perfused with PBS to remove the blood via portal vein.
Cell culture. Human hepatocarcinoma Hep3B cells were cultured in Dulbecco's modified Eagle's medium (DMEM) supplemented with $10 \%(\mathrm{v} / \mathrm{v})$ heat-inactivated foetal bovine serum, penicillin, and streptomycin (Gibco, Carlsbad, CA, USA). Cells were maintained at $37^{\circ} \mathrm{C}$ in a humidified atmosphere containing $5 \% \mathrm{CO}_{2}$. Prior to RNA extraction for expression studies or luciferase assays, cells were treated with bortezomib $(10-250 \mathrm{nM})(14,15)$ in serum-free medium for $15 \mathrm{~h}$. To reduce ER stress, 4-PBA (1-5 mM) was added $1 \mathrm{~h}$ before bortezomib treatment.

Serum enzyme marker measurement. Serum aspartate aminotransferase (AST) and alanine aminotransferase (ALT) levels were measured by the Korean Animal Clinical Research Center (Guri, Korea) using a Hitachi 7020 automatic biochemical analyser (Hitachi, Tokyo, Japan).

Reverse transcription-quantitative PCR (RT-qPCR). Total mRNA from the liver tissues was extracted using an RNeasy Mini kit (Qiagen, Valencia, CA, USA), and cDNA was prepared from the mRNA using a Verso cDNA synthesis kit (Thermo Fisher Scientific, Waltham, MA, USA) according to the manufacturer's instructions. Primer sets are described in Table I. Relative gene expression was calculated as $2^{-\Delta \Delta C t}$ by quantitative PCR, using the SYBR Green PCR Master Mix (Applied Biosystems, Warrington, UK) and an ABI PRISM 7500 Sequence Detection system (Applied Biosystems) (16,17).

Western blot analysis. Liver tissues were lysed in radioimmunoprecipitation assay buffer [50 mM Tris- $\mathrm{HCl}, \mathrm{pH} 7.5,150 \mathrm{mM}$ $\mathrm{NaCl}, 1 \%$ (v/v) Nonidet P-40, $0.5 \%$ (w/v) sodium deoxycholate, $0.1 \%$ (w/v) SDS] containing $50 \mathrm{mM} \mathrm{NaF}, 2 \mathrm{mM} \mathrm{Na}_{3} \mathrm{VO}_{4}$, and protease and phosphatase inhibitors (Sigma-Aldrich). Protein concentration was quantified using Bradford assay reagent (BioRad Laboratories, Hercules, CA, USA). Protein samples $(40 \mu \mathrm{g})$ were subjected to SDS-polyacrylamide gel [8\% (w/v)] electrophoresis and then transferred to nitrocellulose membranes. Membranes were incubated with primary antibodies followed by peroxidase-conjugated secondary antibodies (111-035-003 and 115-035-003; Jackson ImmunoResearch, West Grove, PA, USA) after blocking with $5 \%$ (w/v) BSA in $20 \mathrm{mM}$ Tris- $\mathrm{HCl}$ at $\mathrm{pH} 7.5,500 \mathrm{mM} \mathrm{NaCl}$, and $0.1 \%$ (v/v) Tween-20. The immunocomplexes were detected by chemiluminescence using SuperSignal West Pico chemiluminescent substrate (Thermo Fisher Scientific) and detected with a Bio-Imaging Analyzer (LAS-4000; Fuji, Tokyo, Japan).

Hematoxylin and eosin $(H \& E)$ staining. Liver tissues were fixed in $4 \%(\mathrm{w} / \mathrm{v})$ paraformaldehyde, embedded in paraffin, sectioned at $4-\mu \mathrm{m}$ thickness, and then stained with $H \& E$ using standard methods. Briefly, following deparafinization, the sections were stained with hematoxylin (Sigma-Aldrich) and then destained with acid ethanol ( $1 \mathrm{ml}$ hydrochloric acid $+99 \mathrm{ml} 70 \%$ ethanol). After washing with tap water, sections were stained with eosin (Sigma-Aldrich).

Measurement of GSH levels. Levels of GSH in the fresh liver were measured using a GSH assay kit (Biovision) according to the manufacturer's instructions. Briefly, $40 \mathrm{mg}$ of liver tissues were homogenized with cold glutathione assay buffer, and $6 \mathrm{~N}$ 
Table I. Primers used for quantitative PCR.

\begin{tabular}{lll}
\hline Species & Gene & \multicolumn{1}{c}{ Primer sequences $\left(5^{\prime} \rightarrow 3^{\prime}\right)$} \\
\hline Mouse & CYP2E1 & F: GTTGCCTTGCTTGTCTGGAT \\
& R: AGGAATTGGGAAAGGTCCTG \\
& F: TCATCGGACGCACTTGGAA & R: CAACCACCTTGAATGGCAAGA \\
CHOP & F: GTCCCTAGCTTGGCTGACAGA \\
& R: TGGAGAGCGAGGGCTTTG \\
CX32 & F: TGGTCCCTGCAGCTTATCTT \\
& R: CCTCAAGCCGTAGCATTTC \\
CX43 & F: ATCCAAAGACTGCGGATCTC \\
& R: GACCAGCTTGTACCCAGGAG \\
GAPDH & F: CACTCTTCCACCTTCGATGC \\
& R: CCCTGTTGCTGTAGCCGTAT
\end{tabular}

CYP2E1, cytochrome P450 2E1; BiP, binding immunoglobulin protein; CHOP, CCAAT-enhancer-binding protein homologous protein; CX32, connexin 32; CX43, connexin 43.

perchloric acid was added. The samples were then precipitated with $3 \mathrm{~N}$ potassium hydroxide, and then centrifuged for $2 \mathrm{~min}$ at $13,000 \mathrm{x}$. The neutralized samples were incubated with the $o$-phthalaldehyde probe and glutathione assay buffer for $40 \mathrm{~min}$ to detect reduced GSH. Fluorescence was deteced using a microplate fluorescence reader (BioTek Synergy H1; BioTek, Winooski, VT, USA).

Measurement of CYP2E1 enzyme activity. In order to measure CYP2E1 activity, the rate of transformation of $p$-nitrophenol to $p$-nitrocatechol was analysed with isolated hepatic microsomes, as described previously $(18,19)$. Briefly, the liver microsomes were incubated at $37^{\circ} \mathrm{C}$ in assay buffer containing potassium phosphate buffer (50 mM, pH 7.4), NADPH (1 mM) and $p$-nitrophenol $(0.1 \mathrm{mM})$, at the final concentrations indicated. After $20 \mathrm{~min}$, the reactions were terminated by the addition of $0.6 \mathrm{~N}$ perchloric acid, and centrifuged at $10,000 \mathrm{x} \mathrm{g}$ for $5 \mathrm{~min}$. Subsequently, $6 \mathrm{~N}$ sodium hydroxide was added to the supernatant, and the absorbance was measured at $546 \mathrm{nM}$ using a microplate reader (BioTek Synergy H1; BioTek).

Reporter plasmid construct containing CYP2E1 gene promoter. In the present study, a 540-bp human CYP2E1 gene promoter using 5'-TAGGTACCCAGAAGTGAGATTCC TGTTCT-3' and 5'-CCCAAGCTTTGCCGATGGGGCTCC ACTCT-3' as primers was subcloned into the corresponding restriction sites of the luciferase reporter pGL3-basic vector (Promega, San Luis Obispo, CA, USA), as described in a previous study (20). The underlined letters denote the corresponding restriction sites, KpnI and HindIII The subcloned sequences were verified by DNA sequence analyses.

Luciferase assay. The luciferase assay was performed with the Dual-Luciferase Reporter assay system (Promega) as previously described (21). Reporter plasmid containing the CYP2E1 promoter region was transfected into Hep3B cells using Metafectene transfection reagent (Biontex, San Diego, CA, USA USA), and pRL-cytomegalovirus promoter (Renilla luciferase vector; Promega) was co-transfected to normalise the transfection efficiency. If necessary, pcDNA5/FRT/HNF-1 $\alpha$, obtained from Addgene (Addgene plasmid 31104), or its control vector pcDNA5/FRT/TO was transfected. After $24 \mathrm{~h}$, each well was treated with bortezomib or 4-PBA, as indicated in the figure legends. Cells were lysed using Promega lysis buffer (Promega). Firefly and Renilla luciferase activities were measured sequentially in the same sample using a GloMax $^{\mathrm{TM}}$ 20/20 Luminometer (Promega). Luciferase activities were normalised by dividing the firefly luciferase activity by the Renilla luciferase activity.

Statistical analysis. Values are expressed as the means \pm SEM. Statistical significance was determined using the Student's $\mathrm{t}$-test, and a $\mathrm{p}$-value $<0.05$ was considered to indicate a statistically significant difference.

\section{Results}

Bortezomib treatment alleviates APAP-induced hepatotoxicity in a time-and dose-dependent manner. In order to determine the effect of bortezomib on DILI induced by APAP, we injected bortezomib intraperitoneally twice: first at different time points $(1 \mathrm{mg} / \mathrm{kg})$ (as indicated in Fig. 1A) and then at $1 \mathrm{~h}(1 \mathrm{mg} / \mathrm{kg})$ prior to the administration of APAP. Injecting $500 \mathrm{mg} / \mathrm{kg}$ APAP markedly elevated serum AST and ALT (Fig. 1). When bortezomib was injected $2 \mathrm{~h}$ before APAP administration, serum AST and ALT levels were greatly elevated, similar to the DMSO-treated group (Fig. 1A). Treatment with bortezomib for more than $3 \mathrm{~h}$ was needed to diminish the APAP-induced hepatotoxicity, as indicated by decreased serum AST and ALT levels and H\&E staining (Fig. 1A and C). In addition, bortezomib decreased APAP-induced hepatotoxicity in a dose-dependent manner, and this protective effect was noted at concentrations greater than $0.063 \mathrm{mg}$ bortezomib/kg (Fig. 1B and D).

Bortezomib treatment diminishes hepatotoxicity induced by other chemicals. Since the toxic mechanism differs between 

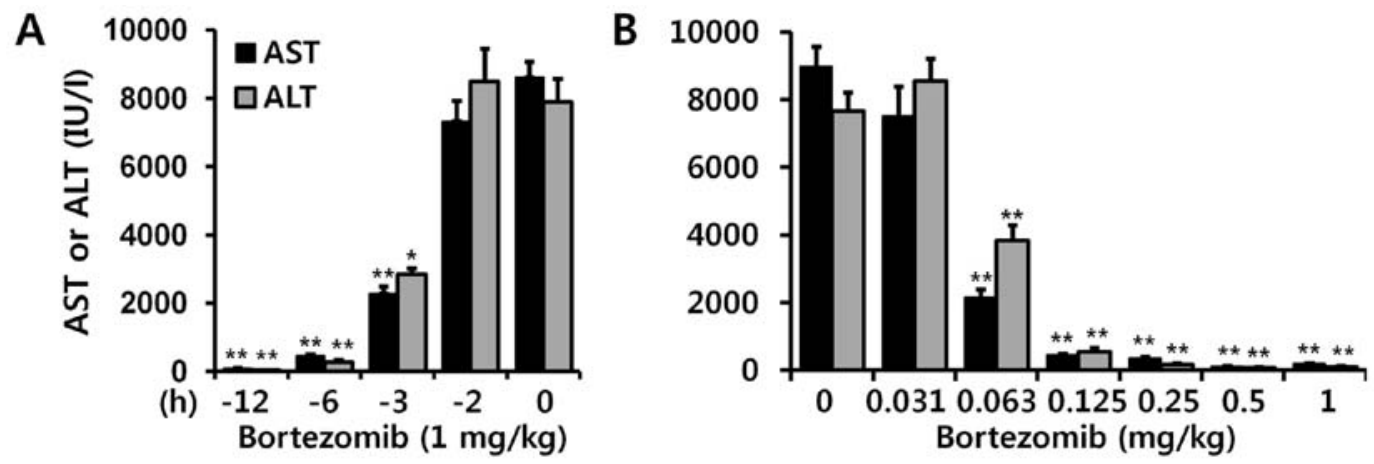

\section{Bortezomib (1 mg/kg)}

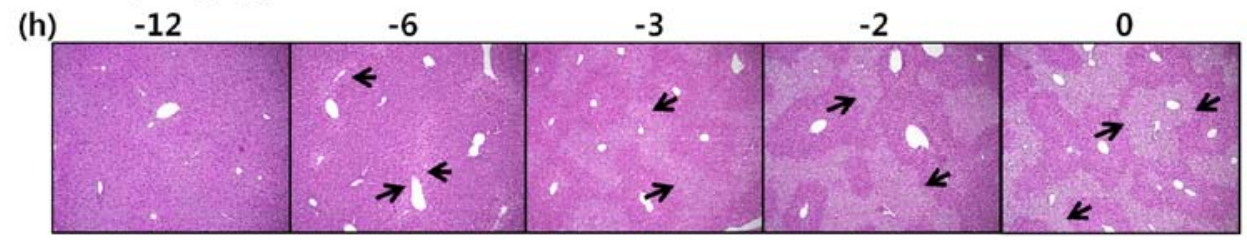

\section{Bortezomib $(\mathrm{mg} / \mathrm{kg})$}

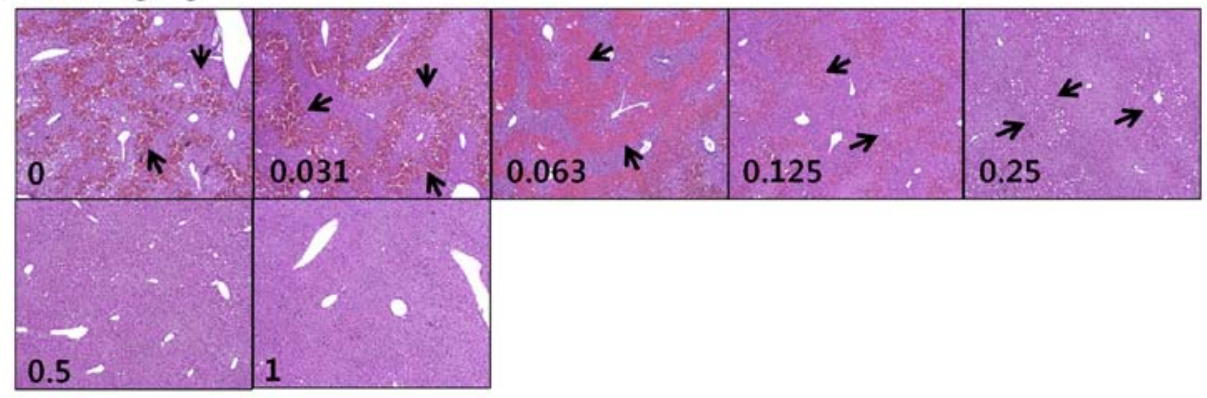

Figure 1. Bortezomib alleviates acetaminophen (APAP)-induced hepatotoxicity in a time- and dose-dependent manner. Bortezomib $(1 \mathrm{mg} / \mathrm{kg}) \mathrm{was}$ intraperitoneally injected twice: first at the indicated time points and then at $1 \mathrm{~h}$ prior to the administration of APAP $(500 \mathrm{mg} / \mathrm{kg})$. The mice were sacrificed $6 \mathrm{~h}$ after the APAP injection. (A) Serum aspartate aminotransferase (AST) and alanine aminotransferase (ALT) levels and (C) H\&E staining of liver sections are shown, and arrows indicate necrotic areas. Various doses of bortezomib $(0-1 \mathrm{mg} / \mathrm{kg})$ were injected twice: first at $12 \mathrm{~h}$ and then at $1 \mathrm{~h}$ prior to the administration of APAP, and (B) serum AST and ALT levels and (D) H\&E-stained liver sections are shown. Data are expressed as the means \pm SEM (n=6). Image magnification, $x 100$. The image is representative of six independent experiments. ${ }^{*} \mathrm{p}<0.05,{ }^{* *} \mathrm{p}<0.01$.

various drugs and chemicals, we investigated whether bortezomib treatment also protects against liver damage caused by other chemicals. Surprisingly, bortezomib treatment also alleviated $\mathrm{CCl}_{4}$ - and TAA-induced hepatotoxicity (Fig. 2). Thus, we posit that the common toxic mechanism of all three chemicals involves CYP2E1 (22-24) or gap junction function (25).

Bortezomib decreases CYP2E1 enzyme expression and activity. GSH is an important antioxidant that detoxifies NAPQI by conjugation, and GSH depletion leads to toxic cascades of NAPQI $(3,4)$. Basal levels of GSH did not differ significantly between DMSO- and bortezomib-treated mice (Fig. 3A). Although GSH was markedly decreased in both DMSO- and bortezomib-treated groups following APAP administration, GSH levels remained higher at all time-points in bortezomib- versus DMSO-treated animals (Fig. 3A). Lower GSH depletion in bortezomib-treated mice implies the decreased formation of NAPQI. Since NAPQI is transformed by CYP2E1, we subsequently measured CYP2E1 expression by both RT-qPCR (Fig. 3B) and western blot analysis (Fig. 3C). Bortezomib treatment significantly decreased CYP2E1 transcription (Fig. 3B), which resulted in lower CYP2E1 protein levels (Fig. 3C). In addition, CYP2E1 enzymatic activity was significantly lower in the livers of bortezomib-treated versus control mice (Fig. 3D). Therefore, we conclude that the decreased CYP2E1 enzyme activity caused by bortezomib administration alleviates liver damage induced by APAP, $\mathrm{CCl}_{4}$, and TAA exposure. This is in agreement with results of previous studies, in which a CYP2E1 deficiency prevented hepatotoxicity induced by these three chemicals (22-24).

Bortezomib diminishes hepatocyte nuclear factor (HNF)-1 $\alpha$-induced CYP2E1 transcription. In order to confirm whether bortezomib regulates CYP2E1 transcription, we transfected a hepatocyte cell line (Hep3B cells) with the reporter plasmid containing the CYP2E1 gene promoter region, and performed luciferase assays. Bortezomib treatment significantly decreased CYP2E1 promoter activity by itself (Fig. 4A) as well as HNF-1 $\alpha$-induced CYP2E1 promoter activity (Fig. 4B). HNF- $1 \alpha$ is the main transcription factor which positively regulates CYP2E1 gene transcription (26-28).

Bortezomib induces endoplasmic reticulum (ER) stress in the liver, which partially contributes to decreased CYP2E1 levels. Since bortezomib can lead to ER stress in vitro $(29,30)$, which can induce decreased CYP2E1 mRNA levels (31), we next 

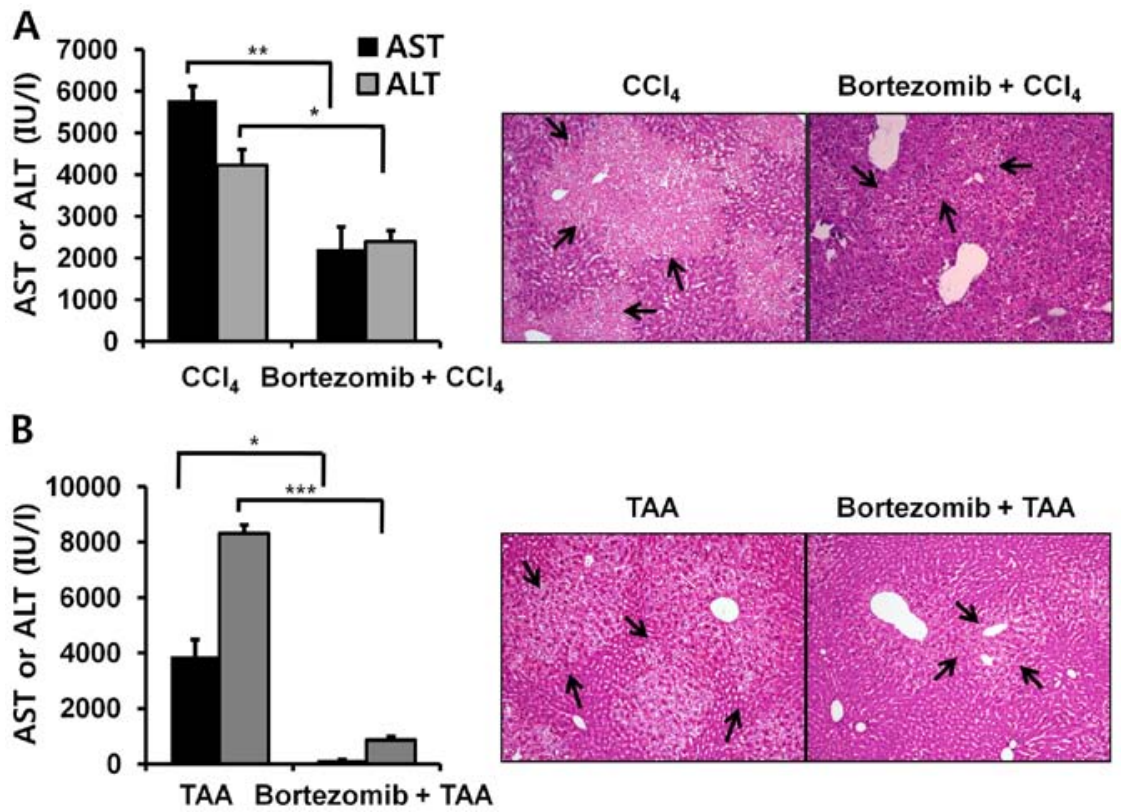

Figure 2. Protective effects of bortezomib on $\mathrm{CCl}_{4}$ - and thioacetamide (TAA)-induced hepatotoxicity. Bortezomib (1 mg/kg) was intraperitoneally injected twice: first at $12 \mathrm{~h}$ and then at $1 \mathrm{~h}$ before $\mathrm{CCl}_{4}(2 \mathrm{ml} / \mathrm{kg})$ or TAA $(200 \mathrm{mg} / \mathrm{kg})$ treatment. The mice were sacrificed $24 \mathrm{~h}$ after the CCl $\mathrm{Cr}_{4}$ TAA injection. (A) Serum aspartate aminotransferase (AST), alanine aminotransferase (ALT) levels, and H\&E-stained sections of liver are shown for the $\mathrm{CCl}_{4}$-injected group. (B) Serum AST, ALT levels, and H\&E-stained liver sections are shown for the group injected with TAA. Data are expressed as the means $\pm \operatorname{SEM}(n=4) .{ }^{*} \mathrm{p}<0.05,{ }^{* *} \mathrm{p}<0.01$, ${ }_{* * * *} \mathrm{p}<0.001$. Image magnification, $\mathrm{x} 100$. Arrows indicate necrotic areas. The image is representative of four independent experiments.
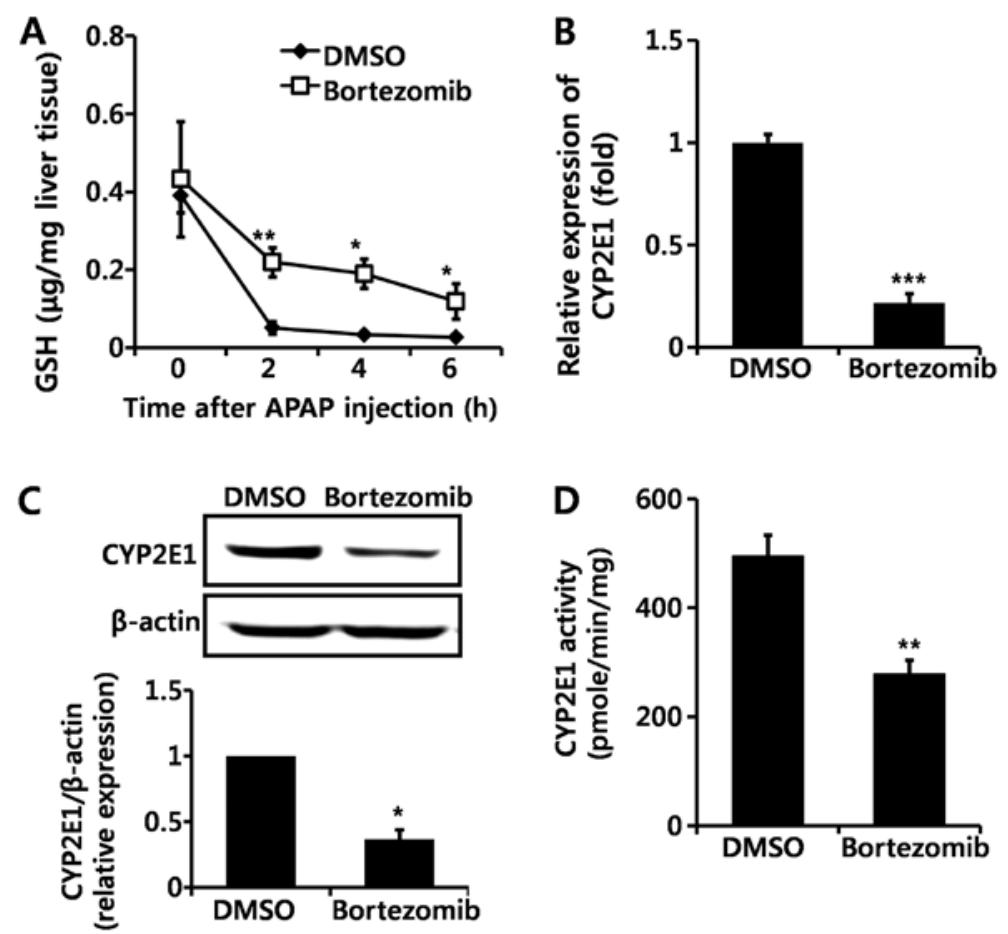

Figure 3. Bortezomib decreases cytochrome P450 2E1 (CYP2E1) expression and enzyme activity. (A) Glutathione (GSH) levels were measured at the indicated times after acetaminophen (APAP) $(500 \mathrm{mg} / \mathrm{kg}$ ) injection. (B) RT-qPCR of hepatic CYP2E1 gene expression and (C) representative western blot analysis (top panel) and quantification (bottom panel) $(\mathrm{n}=6)$ of CYP2E1 levels were examined $12 \mathrm{~h}$ after bortezomib $(1 \mathrm{mg} / \mathrm{kg}$ ) administration. (D) Hepatic CYP2E1 enzyme activity was measured $12 \mathrm{~h}$ after bortezomib $(1 \mathrm{mg} / \mathrm{kg})$ treatment $(\mathrm{n}=6)$. Values are expressed as the means $\pm \mathrm{SEM}(\mathrm{n}=6) .{ }^{*} \mathrm{p}<0.05,{ }^{, * *} \mathrm{p}<0.01,{ }^{* * * *} \mathrm{p}<0.001 . \mathrm{Data}$ are representative of six independent experiments that provided similar results.

investigated whether bortezomib administration also causes ER stress in the liver in vivo. We examined altered levels of several proteins that were reported to be induced during ER stress. BiP, also referred to as glucose-regulated protein 78 , is a central regulator of ER stress (32), and CHOP is one of the most highly induced transcription factors during ER stress (33). Bortezomib 


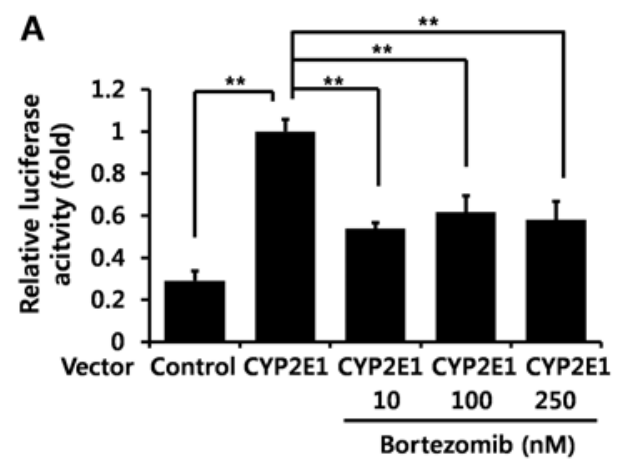

B

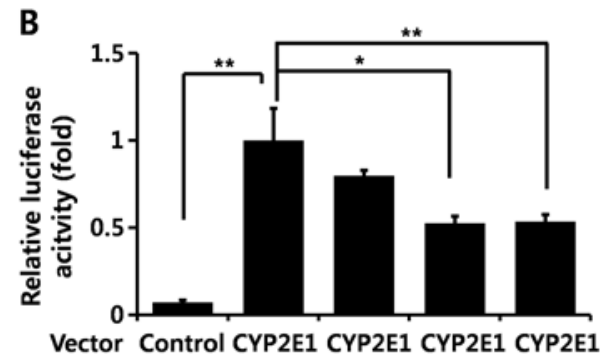

HNF-1 $\alpha$ - ++++

\begin{tabular}{lll}
10 & 100 & 250 \\
\hline Bortezomib (nM)
\end{tabular}

Figure 4. Bortezomib diminishes cytochrome P450 2E1 (CYP2E1) promoter activity in vitro. (A and B) Hep3B cells were transfected with a reporter plasmid containing the CYP2E1 promoter region with or without hepatocyte nuclear factor- $1 \alpha(\mathrm{HNF}-1 \alpha)$, and bortezomib at the indicated concentrations was added for $15 \mathrm{~h}$. Relative luciferase activity was calculated as the ratio of firefly luciferase activity to Renilla luciferase activity in the same sample. Values are expressed as the means \pm SEM $(n=4)$. Data are representative of four independent experiments that provided similar results. ${ }^{*} \mathrm{p}<0.05,{ }^{* *} \mathrm{p}<0.01$. injections markedly elevated hepatic mRNA and protein levels of $\mathrm{BiP}$ and $\mathrm{CHOP}$, indicating the in vivo induction of $\mathrm{ER}$ stress (Fig. 5). IRE-1 $\alpha$ protein expression was also increased $12 \mathrm{~h}$ after bortezomib treatment (Fig. 5C). Subsequently, we used 4-PBA to confirm whether ER stress is directly involved in bortezomib-induced CYP2E1 decrement. Treatment with 4-PBA (5 mM), which is reported to decrease ER stress (34), partially recovered CYP2E1 luciferase activity decreased by bortezomib treatment (Fig. 6). Therefore, bortezomib-induced ER stress partially contributes to decreased CYP2E1 levels.

Protective effect of bortezomib on APAP-induced hepatotoxicity is not derived from direct inhibition of the proteasome. In the present study, in order to investigate whether this effect of bortezomib is due to proteasome inhibition, we injected MG132, another well-known proteasome inhibitor, before APAP administration. By contrast to bortezomib treatment (Fig. 7A and B), MG132 aggravated APAP-induced hepatotoxicity, as demonstrated by elevated serum AST and ALT levels (Fig. 7C) and by enhanced H\&E staining (Fig. 7D). These results suggest that the protective effect of bortezomib on APAP-induced hepatotoxicity is not derived from direct inhibition of the proteasome.

Proteasome inhibitor MG132 does not induce hepatic ER stress. Since we noted that MG132, a second proteasome inhibitor, aggravated APAP-induced liver injury, by contrast with bortezomib (Fig. 1), we subsequently examined the effect of MG132 on CYP2E1 expression and ER stress. Unlike treatment with bortezomib, hepatic mRNA levels of CYP2E1 were
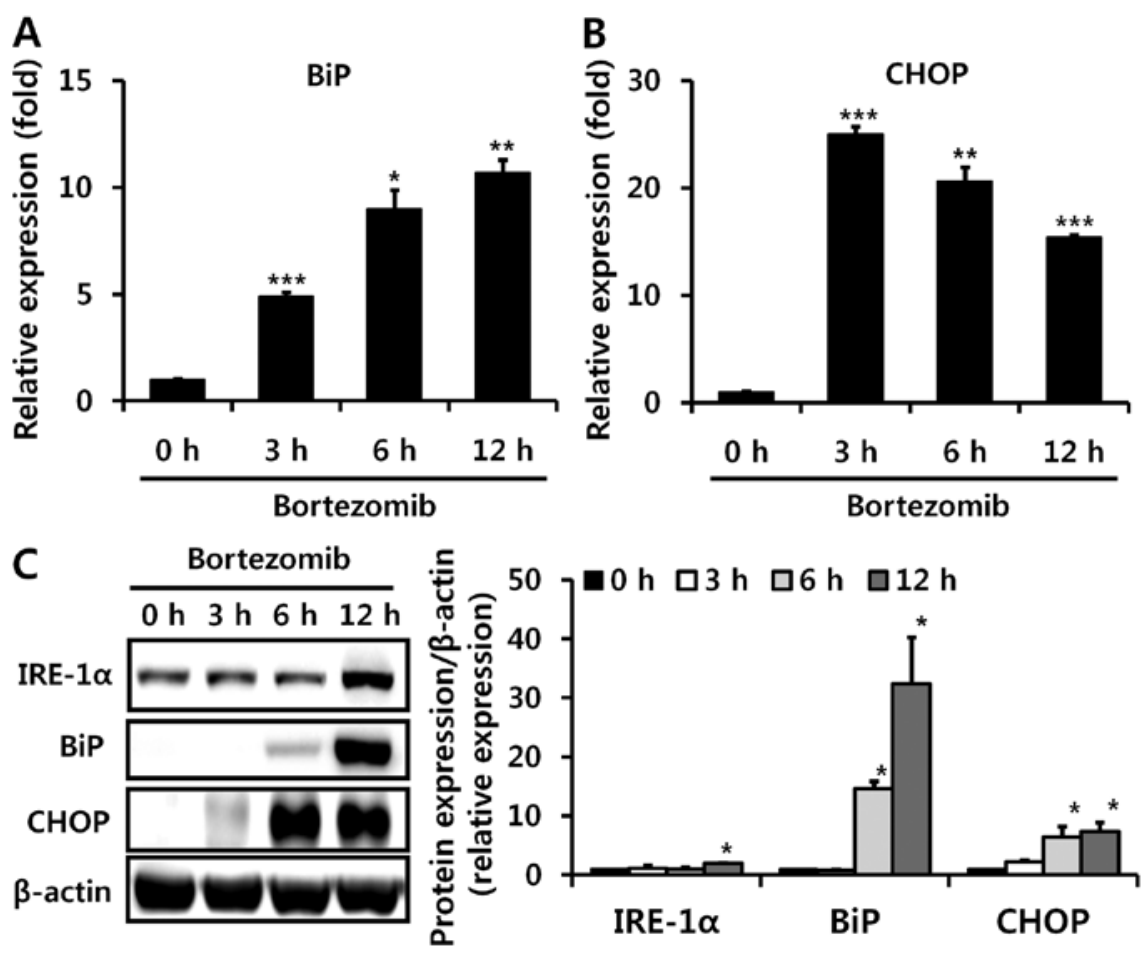

Figure 5. Bortezomib induces endoplasmic reticulum (ER) stress in vivo. Hepatic mRNA levels of (A) binding immunoglobulin protein (BiP) and (B) CCAATenhancer-binding protein homologous protein (CHOP) were measured using RT-qPCR at the indicated times after bortezomib (1 mg/kg) administration. (C) Elevated protein levels of inositol-requiring protein 1 (IRE-1) $\alpha$, BiP, and CHOP by bortezomib administration are shown by western blot analysis (left panel), with results quantified in the right panel $(n=6)$. Values are expressed as the means \pm SEM $(n=6)$. Data are representative of six independent experiments that provided similar results. ${ }^{*} \mathrm{p}<0.05,{ }^{* *} \mathrm{p}<0.01,{ }^{* * * *} \mathrm{p}<0.001$. 


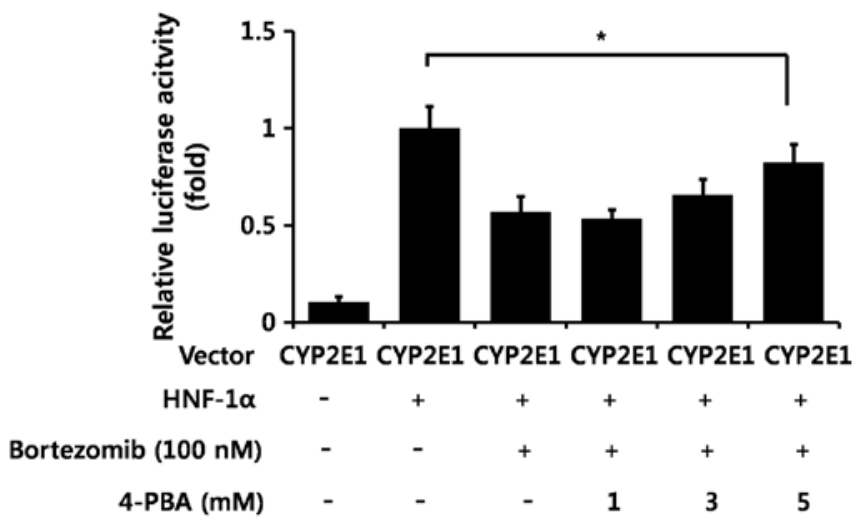

Figure 6. Sodium 4-phenylbutyrate (4-PBA) partially recovered diminished cytochrome P450 2E1 (CYP2E1) promoter activity caused by bortezomib in vitro. Hep3B cells were transfected with a reporter plasmid containing the CYP2E1 promoter region with or without hepatocyte nuclear factor-1 $\alpha$ (HNF-1 $\alpha$ ), and 4-PBA at the indicated concentrations was added for $1 \mathrm{~h}$ before bortezomib treatment. Relative luciferase activity was calculated as the ratio of firefly luciferase activity to Renilla luciferase activity in the same sample. Values are expressed as the means $\pm \operatorname{SEM}(n=4)$. Data are representative of four independent experiments that provided similar results. " $\mathrm{p}<0.05$.

not diminished upon MG132 administration (Fig. 8A), and we also noted relatively unaltered CYP2E1 enzyme activity upon administration of MG132 (Fig. 8B). In addition, it was demonstrated that MG132 administration did not induce ER stress, as indicated by unaltered mRNA levels of BiP and CHOP (Fig. 8C and D).
MG132, but not bortezomib, increases CX32 levels, which increases hepatotoxicity. In addition to CYP2E1 enzyme activity, it has previously been suggested that the common toxic mechanism of all three chemicals (APAP, TAA, and $\mathrm{CCl}_{4}$ ) may also involve gap junctions $(10,25,35,36)$, since gap junctions have been reported to play a critical role in the propagation of hepatotoxicity of all three chemicals used in the present study; ablation of CX32 (a key protein in hepatic gap junctions) significantly protects against DILI $(10,25,35,36)$. Thus, we examined CX32 levels. Notably, MG132 administration significantly increased CX32 protein expression (Fig. 9A), possibly by inhibiting its degradation, and 2-APB, a gap junction inhibitor, abolished MG132-aggravated APAP-induced liver damage (Fig. 9B). Unexpectedly, bortezomib decreased CX32 expression, as well as CX43 mRNA levels (Fig. 9C and D). Therefore, the opposite effects of bortezomib and MG132 on CX32 levels may contribute to the different impacts of bortezomib and MG132 on DILI.

\section{Discussion}

Protein metabolism, including both synthesis and degradation, is crucial for cellular homeostasis. Eukaryotic cells possess three different systems which are necessary for protein degradation: mitochondrial proteases, which degrade the majority of mitochondrial proteins; lysosomal proteases, which degrade membrane and endocytosed proteins; and the ubiquitin-proteasome system, which degrades the vast majority (80-90\%) of intracellular proteins (37). The proteasome system has emerged
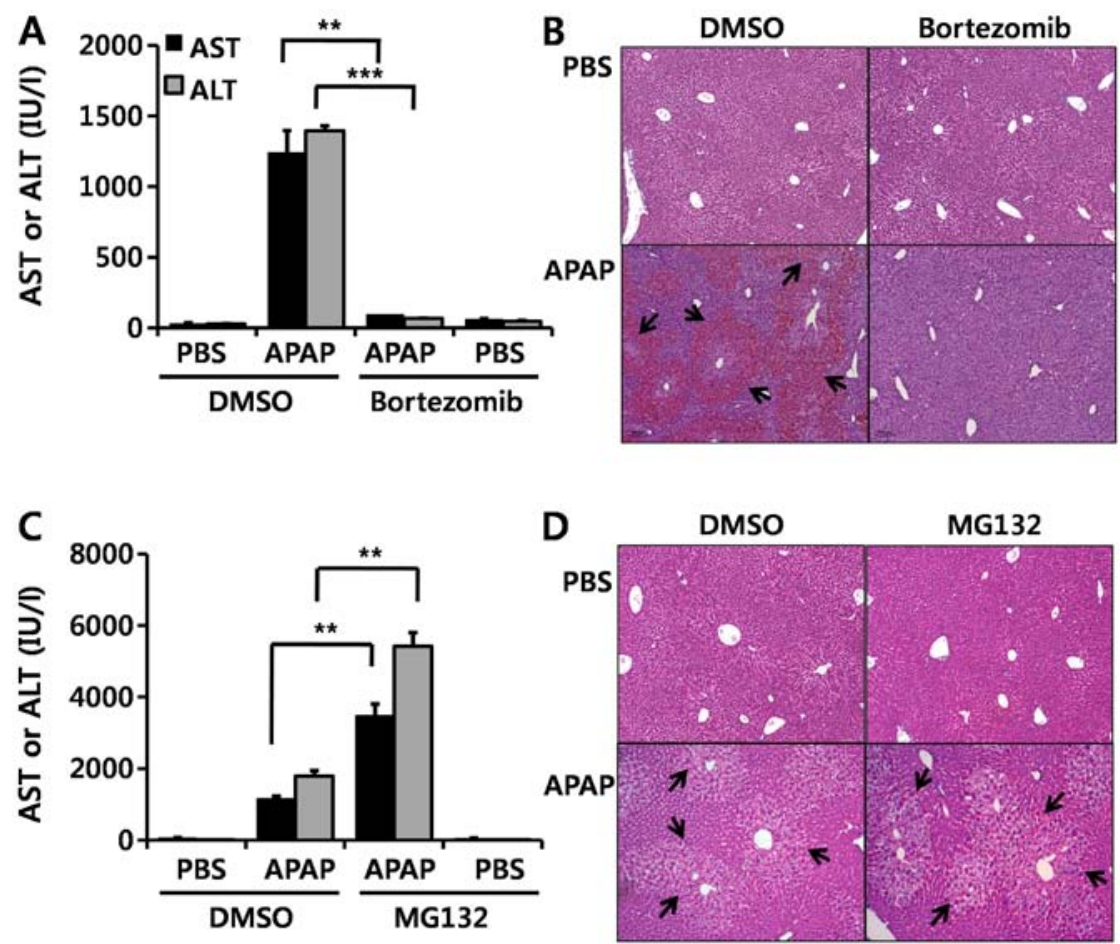

Figure 7. Protective effects of bortezomib on acetaminophen (APAP)-induced hepatotoxicity. Mice were intraperitoneally injected with bortezomib twice: first at $12 \mathrm{~h}(1 \mathrm{mg} / \mathrm{kg})$ and then at $1 \mathrm{~h}(1 \mathrm{mg} / \mathrm{kg})$ before APAP treatment $(350 \mathrm{mg} / \mathrm{kg})$. The mice were sacrificed $6 \mathrm{~h}$ after the APAP injection. (A) Serum aspartate aminotransferase (AST) and alanine aminotransferase (ALT) levels were measured, and (B) hematoxylin and eosin (H\&E)-stained slides of liver sections are shown. Arrows indicate necrotic areas. Similar to bortezomib administration, mice were intraperitoneally injected with MG132 (5 mg/kg) twice: first at $12 \mathrm{~h}$ and then at and $1 \mathrm{~h}$ prior to APAP administration $(350 \mathrm{mg} / \mathrm{kg}$ ). The mice were sacrificed $6 \mathrm{~h}$ after the APAP injection. (C) Serum AST and ALT levels were measured, and (D) liver sections were stained with H\&E, and arrows indicate necrotic areas. Data are expressed as the means $\pm \mathrm{SEM}(\mathrm{n}=5-6)$. ${ }^{* *} \mathrm{p}<0.01$, ${ }^{* * *} \mathrm{p}<0.001$. magnification, $\mathrm{x} 100$. The image is representative of at least five independent experiments. 

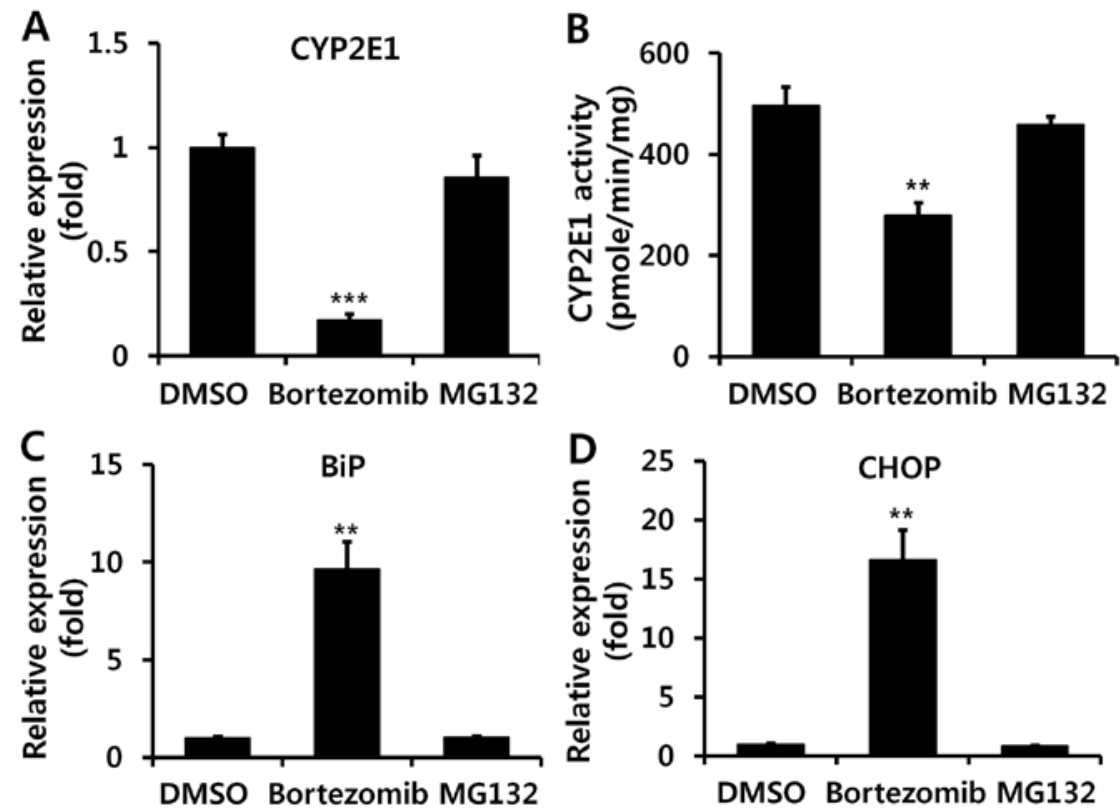

Figure 8. The proteasome inhibitor MG132 does not influence cytochrome P450 2E1 (CYP2E1) enzyme activity. The compound MG132 (5 mg/kg) or bortezomib (1 mg/ $\mathrm{kg}$ ) was injected at $12 \mathrm{~h}$ and $1 \mathrm{~h}$ before liver extraction. (A) Hepatic mRNA levels of CYP2E1 and (B) CYP2E1 enzyme activity were examined $12 \mathrm{~h}$ after bortezomib $(1 \mathrm{mg} / \mathrm{kg})$ or MG132 administration. RT-qPCR analysis of (C) binding immunoglobulin protein (BiP) and (D) CCAAT-enhancer-binding protein homologous protein (CHOP) expression was performed. Values are expressed as the means $\pm \operatorname{SEM}(n=5-6) .{ }^{* *} \mathrm{p}<0.01,{ }^{* * * *} \mathrm{p}<0.001$.

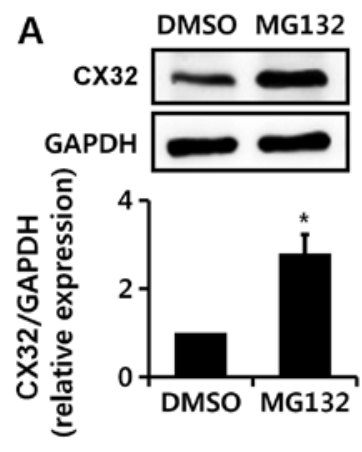

C
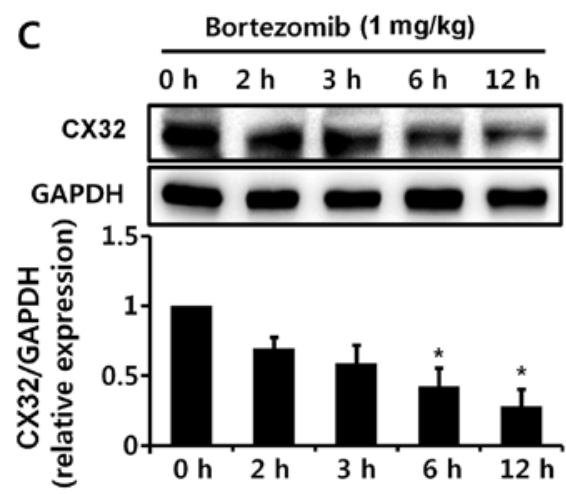

B

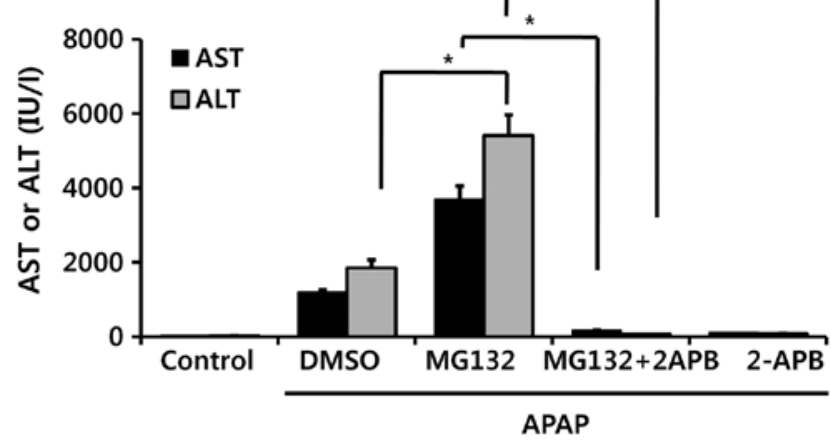

D

$0 \mathrm{~h} 02 \mathrm{~h} \square 3 \mathrm{~h} \square 6 \mathrm{~h} \square 12 \mathrm{~h}$

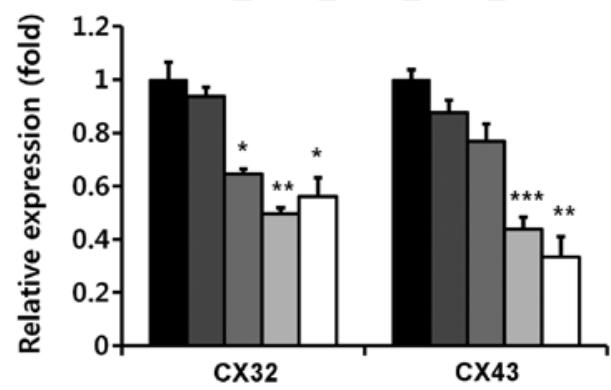

Figure 9. Proteasome inhibitors affect hepatic connexin (CX) 32 levels differently, depending on the drug used. The inhibitor MG132 (5 mg/kg) was injected twice: first at $12 \mathrm{~h}$ and then at $1 \mathrm{~h}$ prior to the administration of APAP. (A) Elevated CX32 protein levels after $12 \mathrm{~h}$ of MG132 treatment are demonstrated by western blot analysis (upper panel), with results quantified in the lower panel ( $\mathrm{n}=6)$. 2-Aminoethyl diphenylborinate (2-APB) (20 mg/kg), a gap junction inhibitor, was injected $1 \mathrm{~h}$ before a second MG132 administration, and APAP was then intraperitoneally injected after $2 \mathrm{~h}$. The mice were sacrificed at $6 \mathrm{~h}$ after the APAP injection. (B) Serum aspartate aminotransferase (AST) and alanine aminotransferase (ALT) levels were measured ( $\mathrm{n}=6$ ). (C) Decreased CX32 protein levels after bortezomib $(1 \mathrm{mg} / \mathrm{kg}$ ) treatment for $12 \mathrm{~h}$ are illustrated by western blot analysis (upper panel), and results are quantified in the lower panel (n=6). (D) RT-qPCR analysis of CX32 and CX43 mRNA was performed at the indicated times after bortezomib administration $(n=6)$. Values are expressed as the means \pm SEM. Data are representative of six independent experiments that provided similar results. ${ }^{*}<<0.05,{ }^{* *} \mathrm{p}<0.01$ and ${ }^{* * *} \mathrm{p}<0.001$.

as a master regulator of diverse cellular processes, including cell cycle, survival, and apoptosis, and plays a critical role in many diseases, such as cancer (38) and neurodegenerative (39) and cardiovascular diseases (37). In the present study, we demon- 
strated that the FDA-approved proteasome inhibitor bortezomib is effective at preventing drug- and chemical-induced acute liver injury via the regulation of CYP2E1 expression.

Hepatic CYP2E1 levels were markedly decreased upon bortezomib treatment. Since CYP2E1 degradation involves the ubiquitin-proteasome pathway $(40,41)$, the protective effect of bortezomib on drug- and chemical-induced liver injury seems attributable to a different mechanism than proteasome inhibition. Although bortezomib was originally developed as a proteasome inhibitor, other distinct mechanisms of bortezomib have been uncovered. One of these is ER stress $(29,30)$, which, in turn, induces IRE-1 $\alpha$-mediated degradation of CYP2E1 mRNA (31). In the present study, ER stress induced by bortezomib treatment also played a role in decreased CYP2E1 transcription, which may alleviate DILI. The main governing factor of CYP2E1 transcriptional regulation is HNF-1 $\alpha$ (26-28), and we noted that bortezomib significantly decreased HNF-1 $\alpha$-induced promoter activation of CYP2E1, and 4-PBA treatment partially recovered bortezomib's inhibition of CYP2E1 promoter activity. Therefore, ER stress plays a role in bortezomib-induced CYP2E1 decrement. Although MG132 was reported to induce ER stress in vitro (42), its administration in vivo did not cause ER stress. Although questions such as whether the relatively long half-life of bortezomib (43) contributes to the different phenotype in vivo compared with MG132, or, rather, a distinct mechanism of bortezomib other than proteasome inhibition may exist, require further study, the protective effect of bortezomib on DILI is noteworthy, considering its current clinical use.

Interestingly, the effects of two well-known proteasome inhibitors, bortezomib and MG132, exerted opposite effects on APAP-induced liver injury. Since proteasome inhibition potentiates CYP2E1-mediated toxicity in HepG2 cells by elevating CYP2E1 levels (44), the aggravating effects of MG132 on APAP-induced liver damage are predictable. However, since no marked change was observed in CYP2E1 enzyme activity, the aggravating effect of MG132 on APAP-induced liver damage appears to be due to some other mechanism, such as gap junction elevation; this was confirmed by elevation of CX32 levels. Gap junctions have recently been implicated as important players in amplifying DILI $(25,36)$. Gap junction channels are composed of CX proteins and play an important role in intercellular communication and in the propagation of liver toxicity and inflammation $(10,25,36)$. Ablation or decreased levels of CX32, a major hepatic gap junction protein, protected against DILI which was induced by the same three hepatotoxic agents used in the present study $(10,25,36)$; thus, diminished CX32 levels caused by bortezomib treatment protect against DILI, and, on the contrary, the increased CX32 levels caused by MG132 are expected to exert opposite effects on DILI. Gap junction proteins are degraded by the proteasome system; thus, proteasome inhibition usually increases CX levels (45-47). On the other hand, ER stress decreases CX43 expression at both the protein and mRNA levels by inhibiting CX43 promoter activity and accelerating the degradation of CX43 (48). Therefore, we suggest that the balance between proteasome inhibition and induction of ER stress determines the effect of bortezomib on CX levels. Decreased bortezomib-induced CX32 levels also contribute to the protective effect on APAP-induced liver injury in addition to decreasing levels of CYP2E1 enzymatic activity.
Since bortezomib is currently being used in clinical settings, the protective effect of bortezomib on APAP-induced liver injury has clinical importance despite its unclear mechanism. Unfortunately, our study shows that bortezomib in mice was only effective when treatments longer than $3 \mathrm{~h}$ were used before APAP administration. Considering that the half-life of CYP2E1 protein is 6-7 $\mathrm{h}(41)$, the time needed for bortezomib to transcriptionally diminish CYP2E1 expression makes sense, but this finding weakens the practical usefulness of bortezomib as a treatment for DILI, since many patients need medical support when liver damage is already present (49). Still, it is possible for bortezomib to be used to prevent DILI, as the use of high doses of some drugs known to be metabolised by CYP2E1 is clinically inevitable. In addition, bortezomib may help to increase the range of drug dosage, which may otherwise be limited due to DILI.

Proteasome inhibition has recently emerged as an effective therapeutic target in several human diseases. The present study suggests that proteasome inhibition has different effects with respect to DILI, depending on the specific drug employed. Bortezomib, but not MG132, was effectively used for alleviating drug- and chemical-induced liver injury in mice. Since ER stress, induced by proteasome inhibition, has distinct effects, each proteasome inhibitor should be individually scrutinised for its therapeutic applicability in future studies.

\section{Acknowledgements}

This study was supported by a Gachon University Research Grant from 2014 (GCU 2014-5101) and awards from the National Research Foundation of Korea funded by the Korean government (Ministry of Education, Science and Technology) (NRF-2013R1A1A1057912).

\section{References}

1. Bernal W, Auzinger G, Dhawan A and Wendon J: Acute liver failure. Lancet 376: 190-201, 2010.

2. Hinson JA, Roberts DW and James LP: Mechanisms of acetaminophen-induced liver necrosis. Handb Exp Pharmacol: 369-405, 2010

3. James LP, Alonso EM, Hynan LS, Hinson JA, Davern TJ, Lee WM and Squires RH; Pediatric Acute Liver Failure Study Group: Detection of acetaminophen protein adducts in children with acute liver failure of indeterminate cause. Pediatrics 118: e676-e681, 2006.

4. Burcham PC and Harman AW: Acetaminophen toxicity results in site-specific mitochondrial damage in isolated mouse hepatocytes. J Biol Chem 266: 5049-5054, 1991.

5. Adams J: The proteasome: structure, function, and role in the cell. Cancer Treat Rev 29 (Suppl 1): 3-9, 2003.

6. Richardson PG, Mitsiades C, Hideshima T and Anderson KC: Bortezomib: proteasome inhibition as an effective anticancer therapy. Annu Rev Med 57: 33-47, 2006.

7. Deming DA, Ninan J, Bailey HH, Kolesar JM, Eickhoff J, Reid JM, Ames MM, McGovern RM, Alberti D, Marnocha R, et al: A Phase I study of intermittently dosed vorinostat in combination with bortezomib in patients with advanced solid tumors. Invest New Drugs 32: 323-329, 2014.

8. Blouin JM, Duchartre Y, Costet P, Lalanne M, Ged C, Lain A, Millet $\mathrm{O}$, de Verneuil $\mathrm{H}$ and Richard E: Therapeutic potential of proteasome inhibitors in congenital erythropoietic porphyria. Proc Natl Acad Sci USA 110: 18238-18243, 2013.

9. Li Z, Wu Q, Yan Z, Li D, Lu G, Mou W, Wu S, Pan X, Lu Q and $\mathrm{Xu} \mathrm{K}$ : The protection and therapy effects of bortezomib in murine acute graft-versus-host disease. Transplant Proc 45: 2527-2535, 2013 
10. Park WJ, Park JW, Erez-Roman R, Kogot-Levin A, Bame JR Tirosh B, Saada A, Merrill AH Jr, Pewzner-Jung Y and Futerman AH: Protection of a ceramide synthase 2 null mouse from drug-induced liver injury: role of gap junction dysfunction and connexin 32 mislocalization. J Biol Chem 288: 30904-30916, 2013.

11. Wagner-Ballon O, Pisani DF, Gastinne T, Tulliez M, Chaligné R, Lacout C, Auradé F, Villeval JL, Gonin P, Vainchenker W and Giraudier S: Proteasome inhibitor bortezomib impairs both myelofibrosis and osteosclerosis induced by high thrombopoietin levels in mice. Blood 110: 345-353, 2007.

12. Carvalho AN, Marques C, Rodrigues E, Henderson CJ, Wolf CR, Pereira P and Gama MJ: Ubiquitin-proteasome system impairment and MPTP-induced oxidative stress in the brain of C57BL/6 wild-type and GSTP knockout mice. Mol Neurobiol 47: 662-672, 2013.

13. Sun H, Kosaras B, Klein PM and Jensen FE: Mammalian target of rapamycin complex 1 activation negatively regulates Polo-like kinase 2-mediated homeostatic compensation following neonatal seizures. Proc Natl Acad Sci USA 110: 5199-5204, 2013.

14. Oerlemans R, Franke NE, Assaraf YG, Cloos J, van Zantwijk I, Berkers CR, Scheffer GL, Debipersad K, Vojtekova K, Lemos C, et al: Molecular basis of bortezomib resistance: proteasome subunit beta5 (PSMB5) gene mutation and overexpression of PSMB5 protein. Blood 112: 2489-2499, 2008

15. Markovina S, Callander NS, O'Connor SL, Kim J, Werndli JE, Raschko M, Leith CP, Kahl BS, Kim K and Miyamoto S: Bortezomib-resistant nuclear factor-kappaB activity in multiple myeloma cells. Mol Cancer Res 6: 1356-1364, 2008.

16. Park JW, Park ES, Choi EN, Park HY and Jung SC: Altered brain gene expression profiles associated with the pathogenesis of phenylketonuria in a mouse model. Clin Chim Acta 401: 90-99, 2009.

17. Livak KJ and Schmittgen TD: Analysis of relative gene expression data using real-time quantitative PCR and the 2(-Delta Delta C(T)) Method. Methods 25: 402-408, 2001.

18. Phillips IR and Shephard EA (eds): Cytochrome P450 Protocols. Humana Press, Totowa, NJ, 2006.

19. Reinke LA and Moyer MJ: p-Nitrophenol hydroxylation. A microsomal oxidation which is highly inducible by ethanol. Drug Metab Dispos 13: 548-552, 1985.

20. Matsunaga N, Ikeda M, Takiguchi T, Koyanagi S and Ohdo S: The molecular mechanism regulating 24-hour rhy thm of CYP2E1 expression in the mouse liver. Hepatology 48: 240-251, 2008

21. Park JW, Lee MH, Choi JO, Park HY and Jung SC: Tissue-specific activation of mitogen-activated protein kinases for expression of transthyretin by phenylalanine and its metabolite, phenylpyruvic acid. Exp Mol Med 42: 105-115, 2010.

22. Wong FW, Chan WY and Lee SS: Resistance to carbon tetrachloride-induced hepatotoxicity in mice which lack CYP2E1 expression. Toxicol Appl Pharmacol 153: 109-118, 1998.

23. Kang JS, Wanibuchi H, Morimura K, Wongpoomchai R, Chusiri Y, Gonzalez FJ and Fukushima S: Role of CYP2E1 in thioacetamide-induced mouse hepatotoxicity. Toxicol Appl Pharmacol 228: 295-300, 2008.

24. Lee SS, Buters JT, Pineau T, Fernandez-Salguero $P$ and Gonzalez FJ: Role of CYP2E1 in the hepatotoxicity of acetaminophen. J Biol Chem 271: 12063-12067, 1996.

25. Patel SJ, Milwid JM, King KR, Bohr S, Iracheta-Vellve A, Li M, Vitalo A, Parekkadan B, Jindal R and Yarmush ML: Gap junction inhibition prevents drug-induced liver toxicity and fulminant hepatic failure. Nat Biotechnol 30: 179-183, 2012.

26. Ueno $\mathrm{T}$ and Gonzalez FJ: Transcriptional control of the rat hepatic CYP2E1 gene. Mol Cell Biol 10: 4495-4505, 1990.

27. Liu SY and Gonzalez FJ: Role of the liver-enriched transcription factor HNF-1 alpha in expression of the CYP2E1 gene. DNA Cell Biol 14: 285-293, 1995.

28. Cheung C, Akiyama TE, Kudo G and Gonzalez FJ: Hepatic expression of cytochrome P450s in hepatocyte nuclear factor 1-alpha (HNF1alpha)-deficient mice. Biochem Pharmacol 66 : 2011-2020, 2003

29. Nawrocki ST, Carew JS, Dunner K Jr, Boise LH, Chiao PJ, Huang P, Abbruzzese JL and McConkey DJ: Bortezomib inhibits PKR-like endoplasmic reticulum (ER) kinase and induces apoptosis via ER stress in human pancreatic cancer cells. Cancer Res 65: 11510-11519,2005.
30. Armstrong JL, Flockhart R, Veal GJ, Lovat PE and Redfern CP: Regulation of endoplasmic reticulum stress-induced cell death by ATF4 in neuroectodermal tumor cells. J Biol Chem 285: 6091-6100, 2010.

31. Hur KY, So JS, Ruda V, Frank-Kamenetsky M, Fitzgerald K, Koteliansky V, Iwawaki T, Glimcher LH and Lee AH: IRE1a activation protects mice against acetaminophen-induced hepatotoxicity. J Exp Med 209: 307-318, 2012.

32. Lee AS: The ER chaperone and signaling regulator GRP78/BiP as a monitor of endoplasmic reticulum stress. Methods 35: 373-381, 2005.

33. Oyadomari S and Mori M: Roles of CHOP/GADD153 in endoplasmic reticulum stress. Cell Death Differ 11: 381-389, 2004

34. Basseri S, Lhoták S, Sharma AM and Austin RC: The chemical chaperone 4-phenylbutyrate inhibits adipogenesis by modulating the unfolded protein response. J Lipid Res 50: 2486-2501, 2009.

35. Tong X, Dong S, Yu M, Wang Q and Tao L: Role of heteromeric gap junctions in the cytotoxicity of cisplatin. Toxicology 310 : 53-60, 2013.

36. Asamoto M,Hokaiwado N, Murasaki T and Shirai T: Connexin 32 dominant-negative mutant transgenic rats are resistant to hepatic damage by chemicals. Hepatology 40: 205-210, 2004.

37. Herrmann J, Ciechanover A, Lerman LO and Lerman A: The ubiquitin-proteasome system in cardiovascular diseases - a hypothesis extended. Cardiovasc Res 61: 11-21, 2004.

38. Mani A and Gelmann EP: The ubiquitin-proteasome pathway and its role in cancer. J Clin Oncol 23: 4776-4789, 2005.

39. Ciechanover A and Brundin P: The ubiquitin proteasome system in neurodegenerative diseases: sometimes the chicken, sometimes the egg. Neuron 40: 427-446, 2003

40. Goasduff T and Cederbaum AI: NADPH-dependent microsomal electron transfer increases degradation of CYP2E1 by the proteasome complex: role of reactive oxygen species. Arch Biochem Biophys 370: 258-270, 1999.

41. Roberts BJ: Evidence of proteasome-mediated cytochrome P-450 degradation. J Biol Chem 272: 9771-9778, 1997.

42. Park HS, Jun Y, Han CR, Woo HJ and Kim YH: Proteasome inhibitor MG132-induced apoptosis via ER stress-mediated apoptotic pathway and its potentiation by protein tyrosine kinase p56lck in human Jurkat T cells. Biochem Pharmacol 82: 1110-1125, 2011.

43. Levêque D, Carvalho MC and Maloisel F: Review. Clinical pharmacokinetics of bortezomib. In Vivo 21: 273-278, 2007.

44. Pérez MJ and Cederbaum AI: Proteasome inhibition potentiates CYP2E1-mediated toxicity in HepG2 cells. Hepatology 37: 1395-1404, 2003

45. Fernandes R, Girão H and Pereira P: High glucose downregulates intercellular communication in retinal endothelial cells by enhancing degradation of connexin 43 by a proteasomedependent mechanism. J Biol Chem 279: 27219-27224, 2004

46. Minogue PJ, Beyer EC and Berthoud VM: A connexin50 mutant, CX50fs, that causes cataracts is unstable, but is rescued by a proteasomal inhibitor. J Biol Chem 288: 20427-20434, 2013.

47. Laing JG, Tadros PN, Westphale EM and Beyer EC: Degradation of connexin43 gap junctions involves both the proteasome and the lysosome. Exp Cell Res 236: 482-492, 1997.

48. Huang T, Wan Y, Zhu Y, Fang X, Hiramatsu N, Hayakawa K, Paton AW, Paton JC, Kitamura M and Yao J: Downregulation of gap junction expression and function by endoplasmic reticulum stress. J Cell Biochem 107: 973-983, 2009.

49. Larson AM: Acetaminophen hepatotoxicity. Clin Liver Dis 11: 525-548, vi, 2007.

50. Farfel-Becker T, Vitner E, Dekel H, Leshem N, Enquist IB, Karlsson S and Futerman AH: No evidence for activation of the unfolded protein response in neuronopathic models of Gaucher disease. Hum Mol Genet 18: 1482-1488, 2009.

51. Hetz C, Lee AH, Gonzalez-Romero D, Thielen P, Castilla J, Soto $\mathrm{C}$ and Glimcher LH: Unfolded protein response transcription factor XBP-1 does not influence prion replication or pathogenesis. Proc Natl Acad Sci USA 105: 757-762, 2008. 